\title{
GW23-e0377 ISCHEAMIC CARDIOMYOPATHY PATIENTS WITH HEART FAILURE-SPECIFIC SERUM PEPTIDOME PATTERN RESEARCH
}

doi:10.1136/heartjnl-2012-302920j.25

Yangyanling Caoxuebin. Baoding City Chinese people's Liberation Army NO.252 Hospital

Objectives The present study provided the basis for the diagnosis of Ischaemic Cardiomyopathy patients, and determined the specific serum peptide profile by comparing the serum differences between Ischaemic Cardiomyopathy (ICM) patients and normal control group. In addition, this study also discuss the function of the difference protein.

Methods The study collected pre-treatment of ICM and normal control serum samples, which were followed by automated MALDI-TOF MS analysis after peptides extracted on magnetic beads coated with WCX phase. Mass spectrographic data was analysed with ClinproTool ${ }^{\mathrm{TM}}$ software, such as GA, SNN and QC. The specific serum peptide model of ICM was established by using genetic algorithms.

Results Through extracting by bead technology with mass spectrometry healthy and disease serum, this study can detect 139 serum peptides. The study detected Ischaemic Cardiomyopathy serum peptidome patterns. The differential expression of the peptide peaks are $47(n=47)$, the study selected $1866.17 \mathrm{Da}$, 2660.54 Da, $7761.49 \mathrm{Da}, 1848.81 \mathrm{Da}, 2037.57 \mathrm{Da}$ peptide peaks, which were statistically significant differences between the two groups, to establish Ischaemic Cardiomyopathy diagnostic model; The 1866.17 Da, 7761.49 Da, 1848.81 Da, 2037.57 Da low expression in the disease group; $600.54 \mathrm{Da}$ high expression in the disease group. The recognition rate, predictive power, sensitivity and specificity of the model were $100 \%$ and $97.49 \%$. One significant difference between the peaks identified by tandem mass spectrometry is the HSP60 protein fragments, it can impede cardiac apoptosis, protect ischaemic myocardial cell microtubules and actin cytoskeleton.

Conclusions The specific serum peptide model had certain applications in the diagnosis and antidiastole of nasopharyngeal carcinoma with heart failure, and provided the basis for discovering a specific marker of Ischaemic Cardiomyopathy. 